\title{
Notas de flora hispánica, VII
}

\author{
María A. Carrasco ${ }^{1}$
}

Resumen: Carrasco, M. A. 2011. Notas de flora hispánica, VII. Bot. Complut. 35: 89-90.

En este trabajo se publican siete taxones de plantas vasculares recolectadas en España y Portugal cuyas citas tienen interés corológico. Entre ellos destaca Sideritis montana, citada en Andalucía occidental únicamente en la provincia de Córdoba y que ahora señalamos en Cádiz; otras tres son primeras citas provinciales y una es la segunda cita provincial. Los pliegos testigo están depositados en el herbario MACB de la Facultad de Ciencias Biológicas de la Universidad Complutense cuyos números de registro se incluyen.

Palabras clave: España, Portugal, Cádiz, Málaga, Toledo, Zaragoza, Algarve, plantas vasculares, corología.

Abstract: Carrasco, M. A. 2011. Notes about Spanish flora, VII. Bot. Complut. 35: 89-90.

In this paper we present seven taxa of vascular plants with chorological interest collected in Spain and Portugal. Four of them are first provincial records and one is the second provincial record. Among them, Sideritis montana has been collected for the first time in Cádiz; this taxon had previously been found only in Cordoba of all Occidental Andalusia. The sheets that testified that are kept in the herbarium of Faculty of Biological Sciences of the University Complutense of Madrid (MACB). The register numbers of the sheets are included.

Key words: Spain, Portugal, Cádiz, Málaga, Toledo, Zaragoza, Algarve, vascular plants, chorology.

\section{INTRODUCCION}

Con esta nota continuamos la serie de aportaciones sobre la distribución de taxones ibéricos, que con este mismo título han ido apareciendo en esta revista. Como en las ocasiones anteriores (Carrasco 1986, 2007; Carrasco \& Martín-Blanco 1995; Martín-Blanco \& Carrasco 1999, 2001; Carrasco et al. 2005), se trata de aportaciones corológicas de interés. Todos los pliegos testigo están depositados en el herbario MACB de la Facultad de Ciencias Biológicas de la Universidad Complutense, y los datos de las etiquetas se transcriben detrás de cada taxón así como el número de registro del herbario. El signo * delante de la provincia marca las primeras citas provinciales.

\section{Sideritis montana L.}

ESPAÑA: *CádIZ: Los Barrios, carretera de Los Barrios a Facinas, taludes y cunetas, 30STF7506, $180 \mathrm{~m}, 31-\mathrm{V}-1996$, M. A. Carrasco, S. Castroviejo \& M. Velayos, 13798SC, MACB101542.
Es la primera cita en Cádiz. En las provincias de Andalucía occidental sólo se señala en Córdoba (Morales 2010: 284-285). Añádase Cádiz a esta distribución.

\section{Senecio adonidifolius Loisel.}

ESPAÑA: ZaRaGoZa: Añón de Moncayo, Monte Altopradillo, en el cortafuegos, 30SXM0224, 1650 m, 20-VI-2002, R. García-Camacho \& M. Bellet, MACB101545.

El mapa de distribución de la especie que presenta Uribe-Echebarría (2005), sólo marca una cita en el límite provincial con Soria.

\section{Luzula campestris (L.) DC.}

ESPAÑA: *TOLEdo: entre El Real de San Vicente y Navamorcuende, junto al área recreativa "El Piélago", praderas junto al río, substrato ácido, 30TUK5245, 1150 m, 23-IV-2004, F. Cabezas, J. M. Cardiel, M. A. Carrasco \& C. Santamaría, FJC 210, MACB 101547.

\footnotetext{
${ }^{1}$ C/ General Pardiñas 118, E-28006 Madrid, España.ma.carrasco@telefonica.net

Recibido: 4 diciembre 2010. Aceptado: 4 enero 2011
} 
Fernández Piedra \& Talavera (2010: 191-192), señalan este taxón en prácticamente todas las provincias limítrofes $(\mathrm{Av}, \mathrm{Cc}, \mathrm{CR}, \mathrm{Cu}, \mathrm{M})$. Añádase To a esta distribución.

Sparganium erectum subsp. neglectum (Beeby) Schinz \& Thell.

PORTUGAL: *ALgarvE: Aljezur, carretera a playa Amoreira, 29SNB1631, 25 m, 08-VI-2001, M. A. Carrasco \& M. Bellet, MACB101548.

Medina (2007: 258), lo señala en las provincias limítrofes (BAI en Portugal y H en España). Añádase Ag a esta distribución.

\section{Allium ursinum L. subsp. ursinum}

ESPAÑA: ZaraGoZa: Litago, Monte de la Mata, cuneta de la carretera, 30SSW0028, 1022 m, 21-VI-2002, R. García-Camacho \& M. Bellet, MACB101544.

Es escasa la presencia del taxón en la provincia (Lorda 2005).

\section{Ornithogalum collinum Guss.}

ESPAÑA: *ToLEDo: Sierra de San Vicente, entre Castillo de Bayuela y El Real de San Vicente, junto a la carretera, substrato ácido, 30TUK5541, 675 m, 23-IV-2004, F. Cabezas, J. M. Cardiel, M. A. Carrasco \& C. Santamaría, MACB 101546.

Citado en la vecina provincia de Cáceres (Paunero 1952: 34, sub O. tenuifolium Guss.). Creemos que esta es la primera vez que se señala en Toledo.

\section{Festuca ampla Hackel subsp. ampla}

ESPAÑA: *MálaGa: Estepona, Sierra Bermeja de Estepona, Los Reales, 30SVF0983, $1400 \mathrm{~m}$, pinares y pinsapares, 15-VI-1994, A. Aparicio, M. A. Carrasco, F. Martin \& M. Velayos, 7438MV, MACB101543.

Aparicio \& Silvestre (1987: 192), lo citan en la limítrofe provincia de Cádiz, señalando que es raro.

\section{AGRADECIMIENTOS}

Agradecemos a Ramón Morales, del Real Jardín Botánico de Madrid (CSIC) su amabilidad al confirmar la identificación de Sideritis montana.

\section{BIBLIOGRAFÍA}

Aparicio, S. \& SilvesTRe, S. 1987. Flora del Parque Natural de la Sierra de Grazalema. Junta de Andalucía. Agencia de Medio Ambiente. Monografías de Medio Ambiente, 5. Sevilla.

Carrasco, M. A. 1986. Notas de flora hispánica. Trab. Dep. Bot. 13: $51-56$.

Carrasco, M. A. 2007. Notas de flora hispánica, VI. Bot. Complut. 31: 97-98.

Carrasco, M. A. \& Martín-Blanco, C. J. 1995. Notas de flora hispánica, II. Bot. Complut. 20: 75-79.

Carrasco, M. A., García-Camacho, R. \& Martín-Blanco, C. J. 2005. Notas de flora hispánica, V. Bot. Complut. 29: 2729.

Fernández Piedra, M. P. \& Talavera, S. 2010. Luzula DC. En: S. Talavera, M. J. Gallego, C. Romero Zarco \& A. Herrero (Eds.), Flora iberica 17: 187-224.

LORDA, M. 2005. M. http://www.ipe.csic.es/floragon/ficha. php?genero=Allium\&especie=ursinum\&subespecie=ursi $\underline{\text { num }}=$ Departamento de Medio Ambiente, Instituto Pirenaico de Ecología y Gobierno de Aragón.

Martín-Blanco, C. J. \& Carrasco, M. A. 1999. Notas de flora hispánica, III. Bot. Complut. 23: 111-113.

Martín-Blanco, C. J. \& Carrasco, M. A. 2001. Notas de flora hispánica, IV. Bot. Complut. 25: 309-312

Medina, L. 2007. Sparganium L. En: S. Castroviejo, M. Luceño, A. Galán, P. Jiménez Mejías, F. Cabezas \& L. Medina (Eds.), Flora iberica 18: 251-258.

MoRALES, R. 2010. Sideritis L. En: R. Morales, A. Quintanar, F. Cabezas, A. J. Pujadas \& S. Cirujano (Eds.), Flora iberica 12: 234-288.

Paunero, E. 1952. Catálogo de plantas recogidas por D. Arturo Caballero en Guadalupe (Cáceres), 1948-1949. Anales Jard. Bot. Madrid 10(1): 25-73.

URIBE-ECHEBARRÍA, P. M. 2005. http://www.ipe.csic.es/flora gon/ficha.php?gener $0=$ Senecio\&especie $=$ adonidifolius $=$. Departamento de Medio Ambiente, Instituto Pirenaico de Ecología y Gobierno de Aragón. 